\title{
Nivel de resiliencia y funcionalidad familiar en adolescentes de un albergue de Ventanilla, Perú
}

\author{
Level of resilience and family functionality in adolescents in a shelter in Ventanilla, Peru \\ Edith Choque Medrano ${ }^{1}$, Hernán Hugo Matta Solis ${ }^{2}$
}

\section{RESUMEN}

Objetivo: Determinar la relación entre el nivel de resiliencia y funcionalidad familiar en adolescentes del albergue de Ventanilla, Perú. Materiales y Métodos: El enfoque del estudio es cuantitativo, el diseño es no experimental, correlacional y de corte transversal. La población estuvo conformada por 160 adolescentes que residen en el albergue. Los instrumentos utilizados fueron escala de resiliencia de Connor-Davidson (CDRISC) y el cuestionario APGAR familiar. Resultados: En relación al nivel de resiliencia, predomino el nivel medio $(52,5 \%)$, seguido del nivel alto $(46,9 \%)$ y bajo $(0,6 \%)$. La dimensión de resiliencia que presento mayor porcentaje de nivel alto de resiliencia fue control y propósito $(61,9 \%)$, la que presento mayor porcentaje de nivel bajo de resiliencia fue espiritualidad $(8,1 \%)$. En cuanto a la funcionalidad familiar predominaron las familias con disfunción leve $(33,1 \%)$, seguido de disfunción moderada $(28,8 \%)$, buena función $(25,6 \%)$ y disfunción severa (12,5\%). La prueba estadística de correlación bivariada de Spearman arroja un coeficiente de correlación de 0,349 ( $\mathrm{p}<0,01$ ), es decir las variables principales del estudio resiliencia y funcionalidad familiar, poseen una relación directamente proporcional moderada con un margen de error de 0,01 (1\%). Conclusiones: El nivel predominante de resiliencia fue el medio. En cuanto a la funcionalidad familiar predominaron las familias con disfunción leve. La variable resiliencia y funcionalidad familiar presentan una relación directamente proporcional moderada.

Palabras clave: Resiliencia; adolescentes; asunción de riesgos; relaciones familiares.

\begin{abstract}
Objectives: To determine the relationship between the level of resilience and family functionality in adolescents of the Ventanilla shelter, Peru. Material and Methods: The study approach is quantitative, the design is non-experimental, correlational and cross-sectional. The population consisted of 160 adolescents residing in the shelter. The instruments used were the Connor-Davidson resilience scale (CD-RISC) and the family APGAR questionnaire. Results: In relation to the level of resilience, the average level predominated $(52.5 \%)$, followed by the high level $(46.9 \%)$ and low level $(0.6 \%)$. The dimension of resilience that presented the highest percentage of high level of resilience was control and purpose $(61.9 \%)$, the one that presented the highest percentage of low level of resilience was spirituality ( $8.1 \%)$. Regarding family functionality, families with mild dysfunction predominated $(33.1 \%)$, followed by moderate dysfunction $(28.8 \%)$, good function $(25.6 \%)$ and severe dysfunction $(12.5 \%)$. The statistical test of bivariate Spearman correlation shows a correlation coefficient of 0.349 ( $\mathrm{p}<0.01$ ), that is, the main variables of the study resilience and family functionality, have a moderate proportional relationship with a margin of error of 0.01 (1\%). Conclusions: The predominant level of resilience was the means. In terms of family functionality, families with mild dysfunction predominated. The variable resilience and family functionality present a directly proportional moderate relationship.
\end{abstract}

Keywords: Resilience; adolescent; risk-taking; family relations.

${ }^{1}$ EP de Enfermería. Universidad de Ciencias y Humanidades. Lima - Perú

\section{INTRODUCCIÓN}

La resiliencia es la capacidad humana para enfrentar, sobreponerse $\mathrm{y}$ ser fortalecido $\mathrm{o}$ transformado por experiencias traumáticas. La definición adoptada por Connor y Davidson, quienes refieren y la definen como un conjunto de cualidades, recursos o fortalezas que favorecen a las personas para afrontar la adversidad (1) y 
logren solucionar sus problemas teniendo una resistencia y adoptando nuevos desafíos.

En la sociedad actual, los problemas de conducta adolescente siguen siendo la preocupación de las familias, frente a este hecho se encuentran con familias capaces de salir adelante conocidas como familia resiliente y otras que se sienten desbordados, sin saber cómo actuar ante situaciones estresantes (agresiones, desobediencias, etc.) (2). Según el reporte por la Organización Mundial de Salud (OMS), refiere que la adolescencia es toda aquella persona que se encuentra dentro de los 10 a 19 años de edad (3), es una etapa en el que la persona se ve enfrentado a una serie de obstáculos, retos uno de los más importantes es su identidad y su independencia familiar, pero sin perder la conexión y pertenencia al grupo. Asimismo, cobra importancia el logro de la madurez biológica, sexual, emocional y social (4). Frente a ello a nivel mundial en el año 2015 murieron cerca de 1,2 millones de adolescentes, es decir, más de 3000 al día. La mayoría de los trastornos de salud mental en la edad adulta comienza a presentarse a partir de los 14 años. Pero en su mayoría no son detectados ni tratados a tiempo (5). Por ello Latinoamérica es conocida como la región más violenta a nivel mundial, donde la violencia forma parte de la vida cotidiana de los niños y adolescentes, en las ciudades grandes donde se observa la violencia y crímenes en los espacios públicos. Este es denominado como la violencia comunitaria. La exposición de armas de fuego, cuchillos, ataques físicos con y sin armas, drogas y delitos aleatorios, tales como el robo y homicidios (6). En el Perú cerca de $81,1 \%$ de adolescentes entre 12 y 17 años, manifiestan que alguna vez en su vida fueron víctimas de violencia psicológica o física. Y alrededor de 8,748 menores de 18 años viven en instituciones o albergues (7), los adolescentes protegidos en los albergues o centros de acogida temporal son más vulnerables y con mayores posibilidades de estar expuestos a abusos de toda índole, incluyendo violencia física y psicológica (8).

La familia es el núcleo fundamental de las sociedades, en ella el ser humano nace, crece y se desarrolla; lo ideal es que en este ambiente natural sus miembros mantengan relaciones interpersonales estables compartiendo y satisfaciendo sus necesidades básicas (8). El concepto de funcionalidad, tuvo su sustento teórico en la propuesta de la Teoría General de Sistemas de Ludwig Von Bertalanffy, una de las características fundamentales de esta teoría es al desarrollo de los sistemas abiertos, los cuales tienen la capacidad de importar y exportar energías o sustancias generando un equilibrio o un estado estable dentro del sistema por lo tanto hacer el sistema funcional (9).

El concepto resiliencia ha ido ganando relevancia en el ámbito de la salud (10), algunas definiciones refieren la resiliencia como un proceso dinámico que se enfoca en la adaptación positiva dentro de un entorno de adversidad (11). Por su parte Grotberg la define como la capacidad universal del ser humano para hacer enfrentar a las adversidades de la vida, poder superarlas o incluso ser transformado por ellas (12). En relación a la Resiliencia como un proceso, se destaca Rutter, quien refiere que la resiliencia se ha caracterizado como un conjunto de procesos sociales e intrapsíquicos que posibilitan tener una vida sana, viviendo en un medio insano (13). Sin embargo, otros autores consideran que la resiliencia está vinculada a las situaciones adversas, siendo un proceso de aprendizaje, cambiante según el contexto, dentro de un continuo vulnerabilidad-resiliencia, asociado a factores de riesgo y de protección (10). Sin embargo, es un hecho que no todo el mundo las afronta de la misma manera, hay personas que se hunden frente a las dificultades, se deprimen, sienten ansiedad, o se estancan en el problema, mientras que otras son capaces no solo de enfrentarse al problema, sino incluso de salir fortalecidas de tales situaciones, se dice que estas personas son «resilientes» (2).

En este estudio la resiliencia es considerada como un conjunto de cualidades personales positivas que facilitan la adaptación individual definido por Connor y Davidson. En el periodo de la adolescencia, la resiliencia implica superar los efectos negativos producidos por la exposición al riesgo mediante estrategias positivas de fortalecimiento y afrontamiento. Durante esta etapa de desarrollo, los adolescentes deberán desplegar una serie de comportamientos resilientes para hacer frente a diversos desafíos y tareas evolutivas dentro del entorno escolar, tales como el incremento de las expectativas académicas y el aprendizaje de comportamientos sociales (14). De modo que la institución formadora forma parte de este proceso en donde se debe promover la resiliencia como una fuente de poder para el estudiante en formación (15).

Como vemos el tema de resiliencia está asociado al desarrollo del adolescente, actualmente vemos que muchos de ellos provienen de hogares con problemas como violencia y disfuncionalidad, esto de hecho afecta su autoestima y adaptación a la sociedad. De no ser identificada esta problemática, estaremos ante un futuro ciudadano con dificultades en su 
proceso de socialización y problemas de salud mental. Tras una revisión de la literatura publicada sobre resiliencia $y$ funcionalidad familiar en adolescentes que residen en albergues, no se han encontrado trabajaos previos que analicen la existencia de combinaciones de las dimensiones resilientes, finalmente revisando la literatura científica, en repositorios nacionales e internacionales, en bases de datos indexadas como Scielo, Science Direct, Scopus y Web of Science, se evidencia que el tema de resiliencia que es tendencia es la propuesta elabora por Connor Davidson, que es poco utilizada en el país. Su propuesta es interesante, ya que las dimensiones que propone se pueden adecuar fácilmente a nuestra realidad. En nuestro país solo se han realizado dos investigaciones utilizando la propuesta e instrumentos de Connor Davidson, la de Cinthya Carpio Huaracha y Eliana Barrios Suarez, que realizaron estudios en internos de medicina y en mujeres quechua hablantes de Ayacucho de 25 a 45 años (16). No se evidencian aun estudios realizados en adolescentes en nuestro país con esta propuesta. Esta es una de las razones de porque se realizó el presente estudio en una población de adolescentes provenientes de familias con dificultades dentro de su estructura y dinámica.

Los resultados son un importante aporte al desarrollo de esta línea de investigación que involucra a los adolescentes cautivos ya sean de escuelas, internados o albergues. Las enfermeras, desde un enfoque de atención centrado en la familia, pueden identificarlas y ayudarlas a normalizar su situación, fomentando la salud familiar y el bienestar de sus miembros. En la actualidad la enfermera está obteniendo competencias formativas de grado y posgrado de gran responsabilidad y especialización en la atención psicosocial. En el campo de la salud mental, para ello disponemos de instrumentos cada vez más refinados científicamente: las taxonomías North American Nursing Diagnosis Association (NANDA), Nursing Intervention Classification (NIC) y Nursing Outcomes Classification (NOC), que facilitan realizar intervenciones enfermeras en el campo de la salud mental (17).

El objetivo fue determinar la relación entre el nivel de resiliencia y funcionalidad familiar en adolescentes del albergue de Ventanilla en Perú.

\section{MATERIALES Y MÉTODOS}

El estudio es de enfoque cuantitativo, en cuanto al diseño es no experimental, correlacional y transversal (18).
La técnica utilizada en este estudio fue la encuesta. Se utilizaron dos instrumentos para medir las dos variables principales. Para la variable resiliencia se utilizó la escala de resiliencia de Connor - Davidson (CD-RISC) (13) el cual mide 5 dimensiones $\mathrm{O}$ factores (Persistencia-tenacidad-autoeficacia, control bajo presión, adaptación y capacidad de recuperarse y espiritualidad), y consta de 25 ítems $(10,11)$. Para la variable funcionalidad familiar se utilizó el cuestionario APGAR Familiar, el cual es útil para evidenciar la forma en que el participante percibe el funcionamiento o dinámica de su familia en un momento dado, mide 5 funciones o dimensiones (adaptación, participación, ganancia, afecto y recursos) a través de 5 ítems (12). Antes de la aplicación de los instrumentos, se coordinó con las autoridades del albergue, para poder desarrollar adecuadamente el trabajo de campo. La población estuvo constituida por 160 adolescentes del albergue. Los criterios de inclusión fueron: Adolescentes cuyas edades estén comprendidas entre 10 a 19 años y tener autorización de sus tutores y la voluntad propia de participar. Se excluyeron a los estudiantes que no eran adolescentes, que no tenían las autorizaciones de sus tutores y los que no se encontraban durante el proceso de recolección de datos por diversos motivos.

Para el análisis de los datos se empleó el paquete estadístico IBM-SPSS Versión 23.0, se utilizaron funciones de la estadística descriptiva como tablas de frecuencias y medidas de tendencia central, entre otras, así como pruebas estadísticas para analizar la correlación entre las variables principales.

En todo el proceso de desarrollo del estudio, se aplicaron los principios de la ética y los criterios de protección de los participantes humanos de la investigación (22). A cada participante, se le envió información a sus padres o tutores y el consentimiento informado, también se indicó a ellos mismos sobre el propósito del estudio, finalmente se les solicitó lean y firmen el asentimiento informado correspondiente.

\section{RESULTADOS Y DISCUSIÓN}

En la Tabla 1 se puede observar los datos generales de los adolescentes de un albergue de Ventanilla, que en total fueron 160. La edad mínima fue 11 años y el valor de la edad máxima fue de 18 años, la edad promedio fue 13,93. De acuerdo al género el masculino estuvo representado por 79 adolescentes que representan el 49,9\%; y el de mayor predominio fue el femenino con 81 que representa el $50,6 \%$. 
Respecto al grado de instrucción la mayoría estaba en el nivel secundario con 127 participantes que representaban el $79,4 \%$, seguido de primaria con 19 participantes que representan el $11,9 \%$ y 14 participantes que representaban el 8,8\% con nivel superior. En cuanto a la procedencia la mayoría de adolescentes provenían de alguna provincia del Perú, siendo estos 116 adolescentes que representan el $72,5 \%$, seguido de 44 participantes que representan el $27,5 \%$ que procedían de Lima.

Tabla 1. Datos generales de los adolescentes de un albergue de Ventanilla, Perú

\begin{tabular}{|c|c|c|}
\hline \multicolumn{3}{|l|}{ Edad } \\
\hline \multirow{2}{*}{ Mínima: 11 Máxima: 18} & \multicolumn{2}{|c|}{ Promedio: 13,93} \\
\hline & $\mathbf{N}$ & $\%$ \\
\hline Totales & 160 & 100 \\
\hline \multicolumn{3}{|l|}{ Sexo } \\
\hline Masculino & 79 & 49,9 \\
\hline Femenins & 81 & 50,6 \\
\hline \multicolumn{3}{|c|}{ Grado de instrucción } \\
\hline Primaria & 19 & 11,9 \\
\hline Secundaria & 127 & 79,4 \\
\hline Superior & 14 & 8,8 \\
\hline \multicolumn{3}{|l|}{ Procedencia } \\
\hline Lima & 44 & 27,5 \\
\hline Provincia & 116 & 72,5 \\
\hline
\end{tabular}

En cuanto al nivel de resiliencia en los adolescentes del albergue de Ventanilla, se puede observar (Tabla 2) que la mayoría de participantes tiene un nivel de resiliencia medio con 84 (52,5\%) adolescentes; seguido de un nivel de resiliencia alto con 75 (46,9\%) adolescentes; y un nivel de resiliencia bajo solo con $1(0,6 \%)$ adolescente.

Tabla 2. Niveles de Resiliencia en adolescentes de un albergue de Ventanilla

\begin{tabular}{lcc}
\hline Resiliencia & N & $\%$ \\
\hline Alto & 75 & 46,9 \\
Medio & 84 & 52,5 \\
Bajo & 1 & 0,6 \\
\hline Totales & 160 & $100 \%$ \\
\hline
\end{tabular}

En cuanto a las dimensiones de la resiliencia (Tabla 3) tenemos que en la dimensión persistencia-tenacidad-autoeficacia, 89 (55,6\%) adolescentes tuvieron un nivel de resiliencia alto, del mismo modo la dimensión adaptación y capacidad de recuperarse, y control y propósito tuvieron un nivel de resiliencia alto con 77 $(48,1 \%) \quad$ y $99 \quad(61,9 \%)$ adolescentes, respectivamente. Sin embargo, las dimensiones Control bajo presión y espiritualidad predomino el nivel de resiliencia medio con $95(59,4 \%)$ y 88 $(55 \%)$ de los participantes respectivamente.

En la Figura 1 se proyecta el comportamiento de la variable funcionalidad familiar en los adolescentes del albergue de Ventanilla; la mayoría de adolescentes presentan una disfunción leve con 53 participantes que representan el $33,1 \%$, seguido de la disfunción moderada con 46 adolescentes que representan el $28,8 \%$, le sigue la buena función familiar con 41 adolescentes que representa el $25,6 \%$ y por último la disfunción severa con 20 participantes que representan el $12,5 \%$.

Figura 1. Funcionalidad familiar en adolescentes de un albergue de Ventanilla $(\mathrm{N}=\mathbf{1 6 0})$

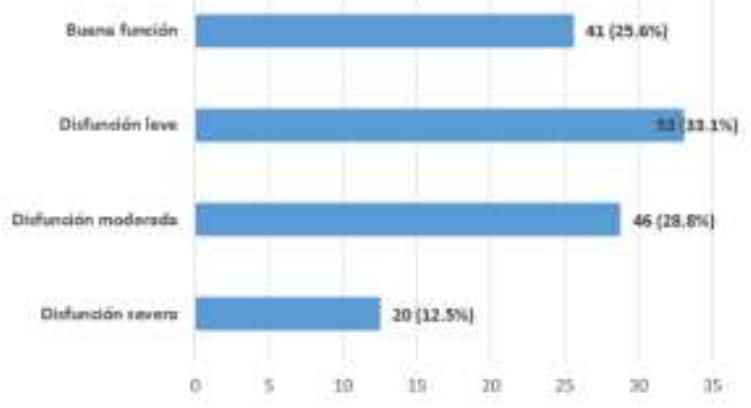

En lo referente a las dimensiones de la funcionalidad familiar en adolescentes del albergue de Ventanilla, el resultado (Tabla 4) en la dimensión Adaptación 53 adolescentes que representan el $33,1 \%$ tienen una disfunción familiar leve, seguido de 46 adolescentes que representan el $28,8 \%$ con disfunción moderada, 41 adolescentes que representan el $25,6 \%$ presentan una buena funcionalidad familiar y solo 20 adolescentes que representan el 12,5\% presentan disfunción severa en esta dimensión. En cuanto a la dimensión participación; 67 de los participantes que representan el 41,9\% tienen una buena funcionalidad familiar, seguida de 44 participantes que representan el $27,5 \%$ con una disfunción familiar moderada, seguido de una disfunción familiar leve con 37 adolescentes que representan el $23,1 \%$ y terminando con una disfunción familiar severa de 12 participantes que representan un 7,5\% de esta dimensión. Del mismo modo la dimensión Ganancia tuvo 60 participantes que representan el $37,5 \%$ con una buena funcionalidad familiar, seguida de 48 que representan el $30 \%$ de los participantes con una disfunción leve, 35 de los participantes que representa el $21,9 \%$ con una disfunción moderada y 17 adolescentes que representan el $10,6 \%$ con disfunción familiar severa en esta dimensión. 
Tabla 3. Dimensiones de la resiliencia en adolescentes de un albergue de Ventanilla $(N=160)$

\begin{tabular}{lcccccc} 
& \multicolumn{2}{c}{ Nivel bajo } & \multicolumn{2}{c}{ Nivel medio } & \multicolumn{2}{c}{ Nivel alto } \\
\cline { 2 - 7 } & $\mathbf{N}$ & $\mathbf{\%}$ & $\mathbf{N}$ & $\mathbf{\%}$ & $\mathbf{N}$ & \% \\
\hline Persistencia- tenacidad- autoeficacia. & 3 & 1,9 & 68 & 42,5 & 89 & 55,6 \\
Control bajo presión & 11 & 6,9 & 95 & 59,4 & 54 & 33,8 \\
Adaptación y capacidad de recuperarse & 10 & 6,3 & 73 & 45,6 & 77 & 48,1 \\
Control y propósito & 10 & 6,3 & 51 & 31,9 & 99 & 61,9 \\
Espiritualidad & 13 & 8,1 & 88 & 55,0 & 59 & 36,9 \\
\hline
\end{tabular}

Tabla 4. Dimensiones de la Funcionalidad familiar en adolescentes de un albergue de Ventanilla (N=160)

\begin{tabular}{|c|c|c|c|c|c|c|c|c|}
\hline \multirow[b]{2}{*}{ Adaptación } & \multicolumn{2}{|c|}{$\begin{array}{l}\text { Disfunción } \\
\text { severa }\end{array}$} & \multicolumn{2}{|c|}{$\begin{array}{l}\text { Disfunción } \\
\text { moderada }\end{array}$} & \multicolumn{2}{|c|}{$\begin{array}{l}\text { Disfunción } \\
\text { leve }\end{array}$} & \multicolumn{2}{|c|}{$\begin{array}{c}\text { Buena } \\
\text { funcionalidad }\end{array}$} \\
\hline & 22 & $13,8 \%$ & 43 & $26,9 \%$ & 29 & $18,1 \%$ & 66 & $41,3 \%$ \\
\hline Participación & 12 & $7,5 \%$ & 44 & $27,5 \%$ & 37 & $23,1 \%$ & 67 & $41,9 \%$ \\
\hline Ganancia & 17 & $10,6 \%$ & 35 & $21,9 \%$ & 48 & $30,0 \%$ & 60 & $37,5 \%$ \\
\hline Afecto & 27 & $16,9 \%$ & 51 & $31,9 \%$ & 37 & $23,1 \%$ & 45 & $28,1 \%$ \\
\hline Recursos & 17 & $10,6 \%$ & 48 & $30,0 \%$ & 33 & $20,6 \%$ & 62 & $38,8 \%$ \\
\hline
\end{tabular}

En la dimensión afecto podemos observar que 51 de los adolescentes que representan el 31,9\% presentan disfunción familiar moderada; seguido de 45 de los adolescentes que representan el $28,1 \%$ con una buena funcionalidad familiar; 37 de los adolescentes que representan el 23,1\% tienen una disfunción familiar leve; seguida de 27 adolescentes que representan el 16,9\% tienen una disfunción familiar severa en esta dimensión. En cuanto a la dimensión Recursos 62 adolescentes que representan el 38,8\% presentan una buena funcionalidad familiar, seguida de 48 adolescentes que representan el $30 \%$ presentan una disfunción familiar moderada; 33 adolescentes que representan el 20,6\% tienen una disfunción familiar leve, seguida de 17 adolescentes que representan el 10,6\% presentan una disfunción familiar severa.

Para determinar el uso de pruebas paramétricas o no paramétricas, se realiza la prueba de normalidad a la distribución de los puntajes de variables "Resiliencia" y "Funcionalidad familiar". La prueba estadística seleccionada (por su pertinencia) es la prueba de KolmogorovSmirnov, la cual mide el nivel de normalidad en la distribución de puntajes en muestras mayores a 30 participantes.

La prueba estadística de correlación bivariada de Spearman arroja un coeficiente de correlación de 0,349 ( $\mathrm{p}<0,01)$ lo que, según el criterio especificado anteriormente, las variables Resiliencia y Funcionalidad familiar, poseen una relación directamente proporcional moderada con un margen de error de 0,01 (1\%).
Tabla 5. Relación entre resiliencia y funcionalidad familiar en adolescentes de un albergue de Ventanilla

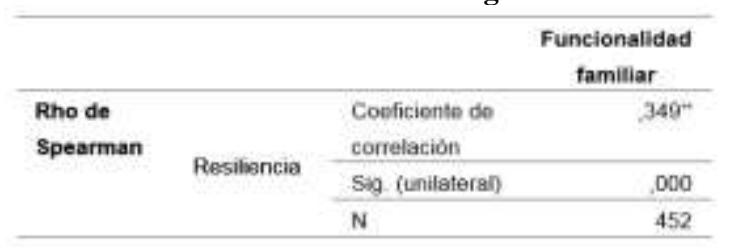

El éxito que pueden lograr en la vida los adolescentes a pesar de haber afrontado adversidades $y$ entornos hostiles, siendo considerados como resilientes, permitió el desarrollo de esta investigación que buscó conocer si existe relación significativa entre la resiliencia y el funcionamiento familiar, quienes en algunos casos a pesar de haber afrontado circunstancias limitantes, demostraron altos niveles de resiliencia, teniendo como uno de sus principales protagonistas para ello a sus familias. Los resultados obtenidos en la presente investigación demuestran que existe relación moderada positiva entre resiliencia y funcionalidad familiar.

Los resultados también se asemejan al estudio realizado por Teófilo Josué García Pérez, Zinia Karen Yupanqui Illacutipa, Katty Puente Guedez, en Lima-Perú, quienes realizaron el estudio "Funcionamiento Familiar y Resiliencia en estudiantes de educación secundaria de una institución educativa pública" en la cual concluyeron que existe relación entre las dimensiones de resiliencia $y$ funcionamiento familiar. Sin embargo los instrumentos utilizados 
para medir ambas variables fueron distintas a la presente investigación (23).

Además se asimilan al estudio realizado por Cajiao Bolaños, María Isabel, en Colombia, quienes realizaron el estudio "Funcionamiento Familiar y Resiliencia en el contexto de exclusión y vulnerabilidad social", en el cual concluyeron que existe relación significativa en sentido positivo entre resiliencia $\mathrm{y}$ funcionamiento familiar (24).

Finalmente es importante resaltar que el estudio servirá para proponer intervenciones oportunas en los adolescentes a nivel de resiliencia y su función familiar. Promover la resiliencia y un buen funcionamiento familiar es un llamado a abordar a un individuo en su totalidad, trabajar con un enfoque multidisciplinario, llevando a la práctica el concepto biopsicosocial. La resiliencia y la funcionalidad familiar demandan una aproximación dinámica que requiere de un enfoque integral y participativo.

\section{CONCLUSIONES}

El nivel predominante de resiliencia fue el medio. En cuanto a la funcionalidad familiar predominaron las familias con disfunción leve. La variable resiliencia $\mathrm{y}$ funcionalidad familiar presentan una relación directamente proporcional moderada.

\section{AUTOR DE CORRESPONDENCIA}

Hernán Hugo Matta Solis

EAP de Enfermería

Universidad de Ciencias y Humanidades

Dirección:

Teléfono:

\section{REFERENCIAS BIBLIOGRÁFICAS}

1. Serrano Parra M, Garrido Abeja M, Notario Pacheco B, Bartolomé Gutierrez R, Solera Martínez M, Martínez Vizcaino V. Validez de la escala de Resiliencia de ConnorDavidson (CD-RISC) en una población de mayores entre 60 y 75 años. International Journal of Psychological [revista en Internet] 2012 [acceso 4 de marzo de 2018]; 5 (2): 4957.

2. Villasana M, Tapia J, Ruiz M. Procesos de afrontamiento $\mathrm{y}$ factores de personalidad como predictores de la resiliencia en adolescentes: validación de un modelo estructural. Revista de Psicodidáctica [internet] 2017 [citado 7 de octubre de 2018]. Universidad de País Vasco; 22(2).

3. Organización Mundial de la Salud. Salud de los adolescentes [Internet]. Ginebra - Suiza: OMS; 2015 [actualizada el 17 de setiembre de 2016; acceso 17 de setiembre de 2016]. [cited 2016 Jan 1].

4. Vinaccia S, Quiceno JM, Moreno E. Resiliencia en adolescentes. Revista Colomibiana de Psicología [Internet] 2007 [acceso 2 de octubre de 2016]; (16): 139-146.

5. Organizacion mundial de salud. Centro de prensa Adolescentes : riesgos para la salud y soluciones [Internet]. Ginebra - Suiza: OMS; 2014 [actualizada el 17 de setiembre de 2016; acceso 17 de setiembre de 2016]. [cited 2016 Sep 17].

6. Mels C., y Fernández L. Violencia comunitaria en adolescentes desfavorecidos: exposición, impacto percibido y consecuencias psicológicas. Revista de Psicología [ Internet] 2015 [acceso 23 de septimbre de 2016]; 24(1): 1- 21.

7. Fondo de las Naciones Unidas para la Infancia. Millones de motivo para seguir trabajando [sede web]. Perú: Unicef;2017 [acceso el 11 de octubre de 2018]. [Internet].

8. Fondo de las Naciones Unidas para la infancia. Protocolo para la atención de la niñez sin cuidado parental en albergues en panamá [sede web] Panamá: Unicef; 2012 [citado el 11 de octubre de 2018]. [Internet].

9. Psicólogos en Madrid EU. Teoría General de Sistemas de von Bertalanffy [sede Web]*. Madrid - España: Psicólogos en Madrid EU; 2017 [actualizado enero de 2017; acceso Septiembre de 2017]. [Internet]. 2013. p. 3.

10. Cabanyes J. Resiliencia: una aproximación al concepto. Revista Psiquiatria y Salud Mental [internet] 2010 [citado 14 de octubre de 2018]; 3(4): 145 - 151.

11. García-Vesga M, Domínguez-De La Ossa E. Desarrollo teórico de la Resiliencia y su aplicación en situaciones adversas: Una revisión analítica. Revista Latinoamericana de Ciencias Sociales, Niñez y Juventud [revista en Internet] 2013 [acceso 4 de marzo de 2018]; 11 (1): 63-77.

12. Echevarría H. La resiliencia/PsicoGuías [sede Web]. Madrid-España: Helena Echevarría; 2017 [actualizado en 2017; acceso en marzo de 2018]. [Internet].

13. Connor K, Davidson J. Development of a new Resilience scale: The Connor-Davidson Resilience scale (CD-RISC). Depression and 
Anxiety [internet] 2003 [citado 14 de octubre de 2018]; 18(2): 76 - 82.

14. Rodríguez A, Ramos E, Ros I, Fernández A, Revuelta L. Resiliencia e implicación escolar en función del sexo y del nivel educativo en educación secundaria. [internet] 2016 [citado el 8 de octubre de 2018]; 44(2):77-82. Instituto de Ciencias de la Educación de la Universidad de Oviedo.

15. Ortunio C, Magaly S, Guevara R, Harold. Aproximación teórica al constructo resiliencia. Comunidad y Salud [internet] 2016 [citado el 7 de octubre de 2018]. 14(2):96-105.

16. Barrios E. Two decades later: The resilience and post-traumatic responses of Indigenous Quechua girls and adolescents in the aftermath of the Peruvian armed conflict. Child Abuse \& Neglect [revista en Internet] 2013 [acceso 4 marzo de 2018]; 37: 200-210. Elsevier Ltd.

17. Serrano M., Garrido M., Notario B., Bartolomé R., Solera M., Martínez V. Validez de la escala de resiliencia de Connor-Davidson(10 ítems) en una población de mayores no institucionalizados. Enferm Clin [Internet]. Elsevier España, S.L.; 2013;23(1):14-21.

18. Hernández Sampieri R., Fernández Collado C., Baptista Lucio M. Metodología de la Investigación. 6a ed. México: McGraw-Hill; 2014. 632 p.

19. Connor K., Davidson J. CDRISC. The Connor-Davidson Resilience Scale [sede Web]. USA: Connor-Davidson; 2018 [actualizado en enero de 2018; acceso 12 de mayo de 2018].

20. Madewell A, Ponce-Garcia E, Martin S. Data replicating the factor structure and reliability of commonly used measures of resilience: The Connor-Davidson Resilience Scale, Resilience Scale, and Scale of Protective Factors. Data in Brief [revista en Internet] 2016 [acceso 18 de mayo de 2018].

21. Suarez M, Alcalá M. Apgar Familiar: Una Herramienta para detectar disfunción familiar. Revista Medica La Paz [revista en Internet] 2014 [acceso 4 de marzo de 2018]; 20 (1): 53-57.

22. Gomez P. Principios básicos de bioética. Revista Peruana de Ginecología y Obstetricia [revista en Internet] 2009 [acceso 8 de febrero de 2018]; 55(4): 230-233.

23. García T. Yupanqui Z. Puente K. Funcionamiento familiar y resiliencia en estudiantes con facultades sobresalientes del tercer año de secundaria de una institución educativa pública con altas exigencias académicas, durante el período 2013. Rev Cient Ciencias la Salud [Internet]. 2014;72(2013):29-34.

24. Cajiao M. Funcionalidad familiar y resiliencia en contexto de exclusión y vulnerabilidad sacial [tesis doctoral]*. Madrid - España: Universidad Pontificia Comillas; 2015. [Internet]. 